\title{
Phase diagram of the two-dimensional quantum electron freezing with external impurities
}

\author{
S. T. Chui \\ Bartol Research Institute, University of Delaware, Newark, Delaware 19716 \\ B. Tanatar \\ Physics Department, Bilkent University, 06533 Ankara, Turkey
}

(Received 4 November 1996)

\begin{abstract}
We discuss the phase diagram of the quantum electron solid-fluid transition in the presence of impurities in zero magnetic fields. This is based on recent improved understanding of the response function in both the fluid and the solid phases. [S0163-1829(97)03816-2]
\end{abstract}

Recently there has been much interest in the low-density limit of two-dimensional (2D) electrons in GaAs heterojunctions in an external magnetic field ${ }^{1}$ and Si-MOSFET's (metal-oxide-semiconductor field-effect transistors) in high and zero field ${ }^{2}$ where a freezing transition to a solid seems to occur as the density is lowered. Monte Carlo (MC) simulations $^{3}$ and analytic calculations ${ }^{4}$ for the pure system at zero magnetic field suggest that the solid-fluid transition occurs near $r_{s} \approx 37$. Here $r_{s}=1 / \sqrt{\pi n} a_{B}$ where $a_{B}=\hbar^{2} \epsilon / m^{*} e^{2}$ is the Bohr radius, $n$ is the density, $m^{*}\left(0.2 m_{e}\right.$ for Si-MOSFET's) is the effective mass, and $\epsilon$ (7.7 for Si-MOSFET's) is the dielectric constant. Pudalov et al. ${ }^{2}$ reported observation of a solid-fluid transition in Si-MOSFET's near $r_{s}=10$. The experimental systems are not perfect. Typically there are dopants located at some distance $d$ (setback distance) away from the quantum well. To confront experiment with theory, we have performed a quantum Monte Carlo simulation ${ }^{5}$ that includes the impurity effects and the Coulomb interaction on the electron solid-fluid transition with parameters appropriate for the Si-MOSFET system investigated by Pudalov et al. ${ }^{2}$ Good agreement with the experimental results is found. A key element in this calculation is the difference of the electron fluid and solid response to the effect of external impurities. Subsequently, we have performed a systematic study of the response function of the electron solid by Monte Carlo simulation. ${ }^{6}$ Thus, we are now in possession of a much more accurate understanding of the response function of both the electron solid and the fluid. ${ }^{7}$ The purpose of this paper is to apply our improved knowledge of the response function to understanding the physics and the systematic trends of the effect of impurities on the quantum electron solid-fluid transition. Our final result is summarized in the phase diagram in Fig. 1 where we show the transition density as a function of the setback distance $d$ of the external charged impurities for three values of their concentration. We now explain our results in detail.

The change in energy due to external impurities is calculated by

$$
\Delta E_{i}=-\frac{1}{2} \sum_{q} V_{i}(q)^{2} \chi(q) / n
$$

where $V_{i}(q)=\left(2 \pi e^{2} / q\right) e^{-q d}$ is the potential for charged impurities at a setback distance $d$ from the 2D electron system. $\chi(q)$ is the static density response function at wave vector $q$. The difference in energy gained from the fluid and the solid phase thus depends on the difference of the response function between these two phases. We first compare the $\chi(q)$ in the solid and fluid phases in Fig. 2. In the solid phase, $\chi(q)$ with $\mathbf{q}$ along the $(1,0)$ direction obtained from the Monte Carlo simulations ${ }^{6}$ is depicted by solid squares. The dashed lines indicate the approximation involving onephonon contribution with the Debye-Waller factor,

$$
\chi_{1}(q+K)=\left[(\mathbf{q}+\mathbf{K}) \cdot \mathbf{e}_{q i}\right]^{2} \exp \left\{-\left\langle[(\mathbf{q}+\mathbf{K}) \cdot \delta \mathbf{r}]^{2}\right\rangle\right\} / m \omega_{q i}^{2},
$$

in which $\mathbf{K}$ is a reciprocal-lattice vector, $\mathbf{e}_{q i}$ is the unit polarization vector, and $\omega_{q i}$ are the harmonic phonon frequencies. $^{8} \quad$ To calculate the Debye-Waller factor $\exp \left\{-\left\langle[(\mathbf{q}+\mathbf{K}) \cdot \delta \mathbf{r}]^{2}\right\rangle\right\}$ we use the approximation ${ }^{9}\left\langle(\delta x)^{2}\right\rangle$ $=\left\langle\delta r^{2}\right\rangle \approx a^{2}\left(40 / r_{s}\right)^{1 / 2} / 16$. In the fluid phase, we employ

$$
\chi(q)=\chi_{0} /\left\{1-v_{q}[1-G(q)] \chi_{0}\right\}
$$

where

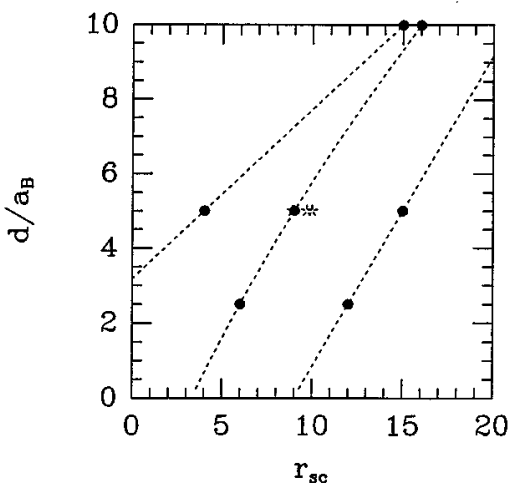

FIG. 1. The phase diagram for the solid-liquid transition in terms of $d$ and $r_{s c}$. The solid circles are our calculated results for different impurity concentrations and setback distances $d$. The dotted lines are guide to the eye at impurity concentrations $n_{i}$ $=10^{9} \mathrm{~cm}^{-2}, n_{i}=10^{10} \mathrm{~cm}^{-2}$, and $n_{i}=10^{11} \mathrm{~cm}^{-2}$ (from top to bottom). The experimental points of Pudalov et al. (Ref. 2) are indicated by stars. 

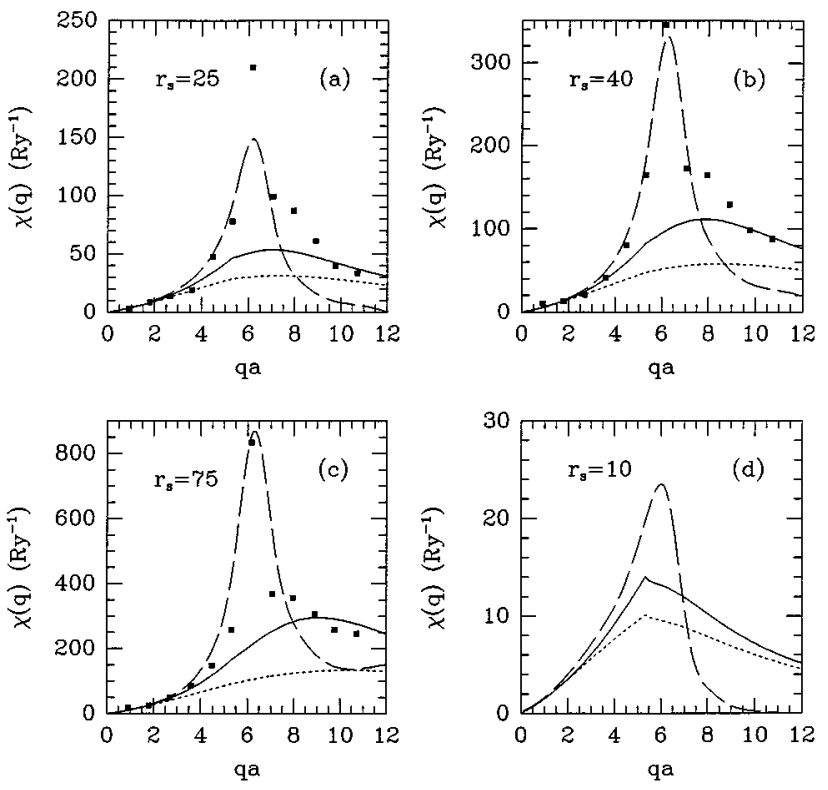

FIG. 2. The static density response function $\chi(q)$ in the solid and fluid phases at (a) $r_{s}=25$, (b) $r_{s}=40$, (c) $r_{s}=75$, and (d) $r_{s}$ $=10$. The solid squares are results of MC simulations (Ref. 2), and the dashed lines are one-phonon approximation for the solid phase. Solid and dotted lines indicate the fluid-response function with and without the local-field effects, respectively.

$$
\begin{gathered}
\chi_{0}=f\left(q / 2 k_{F}\right) / 2 \pi a_{B}^{2} \mathrm{Ry}^{-1}, \\
f(x)=\left[1-\theta(x>1) \sqrt{x^{2}-1} / x\right] .
\end{gathered}
$$

Here $G(q)$ is the local-field factor describing the exchangecorrelation effects not accounted for within the randomphase approximation (RPA). We use the parametrized form ${ }^{10}$ of $G(q)$ that incorporates the correlation energies of accurate Monte Carlo simulations. ${ }^{3}$ The fluid-static response function reduces to the RPA when we set $G(q)=0$, that is shown by the dotted lines. The importance of exchange-correlation effects in the fluid phase can be seen in Fig. 2. More accurate static response function of $2 \mathrm{D}$ electrons in the fluid phase was calculated by Moroni, Ceperley, and Senatore using MC simulations. $\chi_{\text {fluid }}(q)$ with the parametrized $G(q)$ agrees well with the MC results ${ }^{7}$ in the small- and high- $q$ limits, but differences exist in the intermediate- $q$ region. The approximation that we use has the virtue of simplicity. The error created in the energy differences with this approximation is small because the solid response function is much larger than the fluid response function in the intermediate- $q$ region. Thus, the difference between the solid and the more accurate fluid response function is not that different from the difference of the solid and the approximate fluid response function used here.

The noteworthy features of the comparison between the solid and fluid response functions are summarized as follows. In the solid phase, the one-phonon approximation (with the Debye-Waller factor) represents the Monte Carlo results rather well for small $q$ at all densities. In fact, both the solid and fluid $\chi(q)$ have the same long-wavelength limit as may be seen in Fig. 2. At intermediate values of $q$ close to the reciprocal lattice vector, the solid response function is
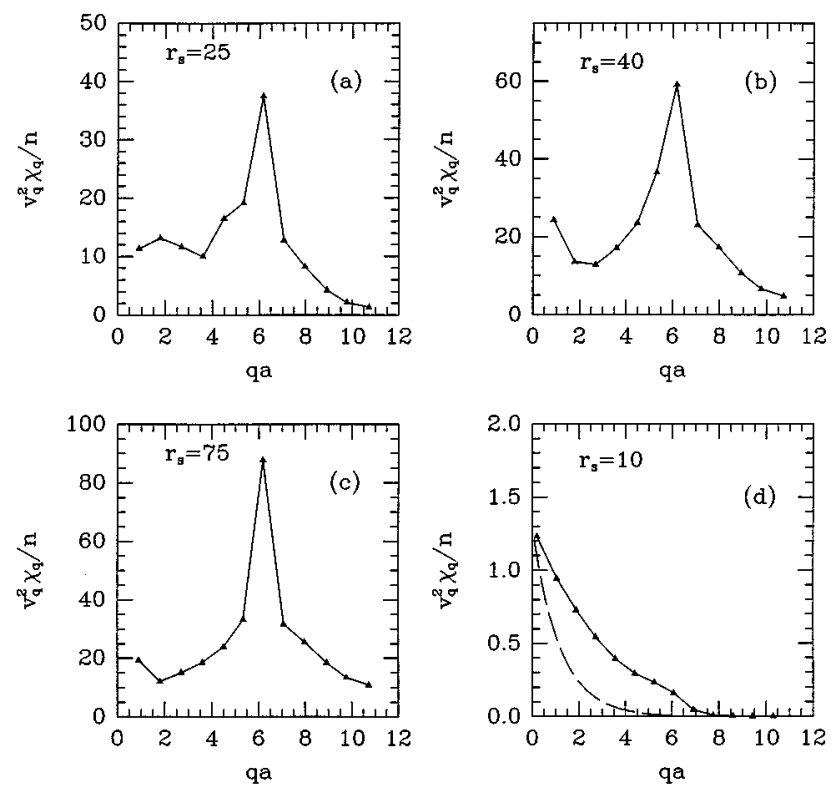

FIG. 3. The integrand $V_{i}(q)^{2} \chi(q) / n$ in the solid phase for $d$ $=5 a_{B}$ at densities (a) $r_{s}=25$, (b) $r_{s}=40$, (c) $r_{s}=75$, and (d) $r_{s}$ $=10$. The solid lines connect the points to guide the eye (no interpolation) and the dashed line for $r_{s}=10$ is the corresponding result for the fluid phase.

much larger than that for the fluid. It is this difference that leads to the possible stabilization of the solid phase. For $q$ larger than this reciprocal-lattice vector, the $\chi_{\text {fluid }}(q)$ (including the local-field corrections) is similar to $\chi_{\text {solid }}(q)$ obtained from Monte Carlo simulations whereas the one-phonon approximation is much smaller. We have ${ }^{6}$ previously estimated the impurity energy using Eq. (1). However, in the solid response function, the Debye-Waller factor was not included. In the fluid response function, only the Hubbard approximation for $G(q)$ was used.

In the formula [Eq. (1)] for the energy, it is the product of the square of the impurity potential and the response function that enters. Since the nature of impurity potential is Coulombic, it is also cut off by the exponential function for $q$ larger than the $1 / d$, the major part of the integral for $\Delta E_{i}$ comes from the region $|q|<1 / d$. Thus, even though the Monte Carlo and one-phonon approximation results for $\chi_{\text {solid }}$ differ considerably at large momenta, insofar as the $\Delta E_{i}$ is concerned, using the one-phonon approximation would not lead to serious errors. Similar arguments will also hold in the fluid phase. This is another reason why our use of model $\chi_{\text {fluid }}(q)$ (with parametrized local-field corrections) as opposed to the MC- $\chi_{\text {fluid }}(q)$ should be a reasonable approximation. We illustrate the integrand $V_{i}(q)^{2} \chi(q) / n$ in Fig. 3 for various densities in the solid phase. As the density decreases (small $r_{s}$ ) so that $d$ becomes much larger than the lattice spacing the weight of the integral completely shifts to the small- $q$ side. Since the small- $q$ response function of the fluid and the solid is the same, the difference in the energy gained from the external impurities becomes smaller the smaller the electron density is.

Our results of the perturbation calculation for the solid and fluid energies in the presence of impurities are given in Fig. 4 for various impurity distances. The open squares de- 

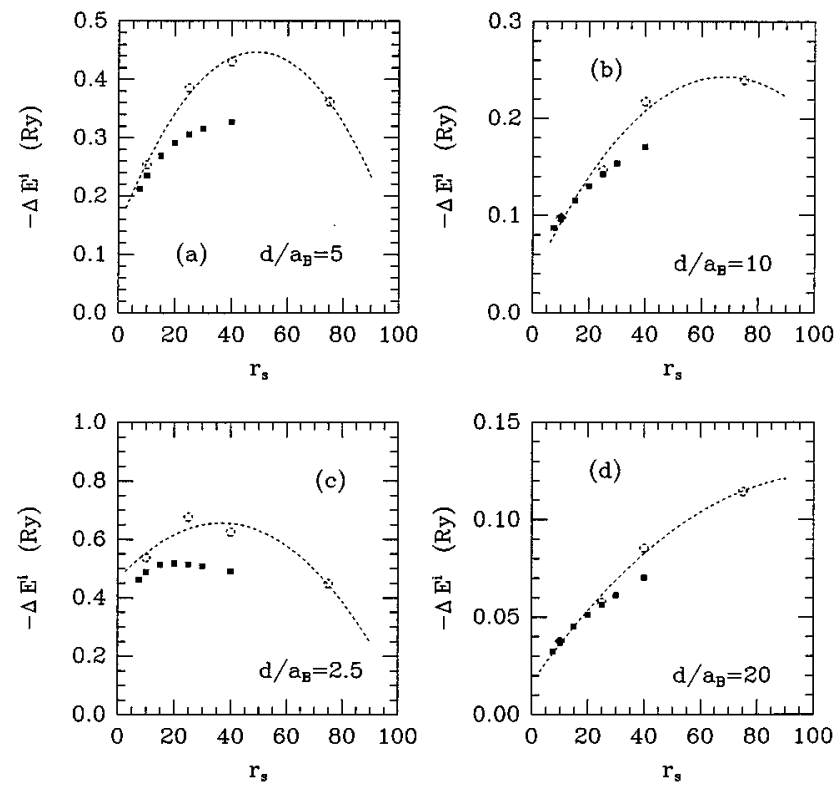

FIG. 4. The change in energy of the electron system due to impurities as a function of $r_{s}$, at (a) $d=5 a_{B}$, (b) $d=10 a_{B}$, (c) $d$ $=2.5 a_{B}$, and (d) $d=20 a_{B}$. The solid and open squares are for solid and fluid phases, respectively. The dotted line is a quadratic fit in the solid phase to guide the eye.

note the solid energies, where the $\mathrm{MC}-\chi_{\text {solid }}(q)$ were used except $^{11}$ at $r_{s}=10$. The dotted line is a quadratic interpolation through the points to guide the eye. The solid squares are for the fluid phase. The total energy difference is the sum of the energy difference without the impurities and $\Delta E_{i}$. Figure 5 shows the difference in fluid and solid energies as a function of $r_{s}$, with and without external impurities. We use the expression

$$
\begin{aligned}
\Delta E\left(r_{s}, d\right)= & E_{\text {fluid }}\left(r_{s}\right)-E_{\text {solid }}\left(r_{s}\right) \\
& +n_{i}\left[\Delta E_{\text {fluid }}^{i}\left(r_{s}, d\right)-\Delta E_{\text {solid }}^{i}\left(r_{s}, d\right)\right],
\end{aligned}
$$

where the first two terms refer to the impurity-free $2 \mathrm{D}$ electron gas, and we have assumed the impurity energy to be proportional to the impurity density $n_{i}$. The presence of impurities at a setback distance $d=5 a_{B}(\simeq 100 \AA)$ shifts the solid-liquid transition in a pure system from $r_{s c} \simeq 37$ to

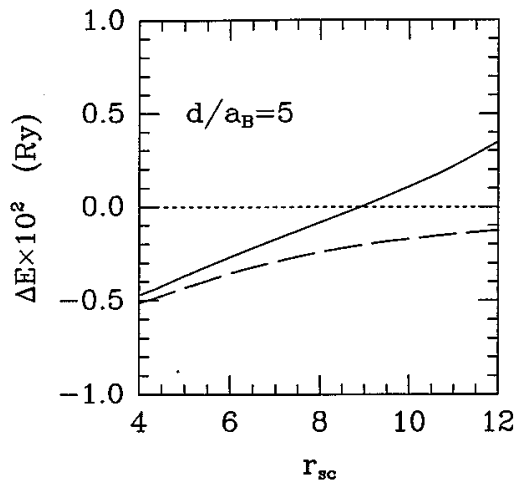

FIG. 5. The energy difference in solid and fluid phases as a function of $r_{s}$ at impurity distance $d=5 a_{B}$. The solid line includes the effects of impurities, whereas the dashed line is for a pure system.

$r_{s c} \simeq 8.5$, consistent with the recent experiments of Pudalov et $a .^{2}$

Finally, we present the phase diagram of a $2 \mathrm{D}$ electron system with impurities in Fig. 1. Impurity distance against the critical density $r_{s c}$ at which the solid-liquid transition occurs is shown for fixed impurity concentration $n_{i}$. In the experiments of Pudalov et al. ${ }^{2}$ three samples of differing mobilities (impurity concentrations) were used. Assuming that the observed peak mobilities are proportional to $n_{i}$ and carrying out an analysis similar to that given by Gold ${ }^{12}$ we infer that the samples of Pudalov et al. ${ }^{2}$ have critical densities $n_{c}$ $=8.9 \times 10^{10} \mathrm{~cm}^{-2}, 9.7 \times 10^{10} \mathrm{~cm}^{-2}$, and $10^{10} \mathrm{~cm}^{-2}$. We indicate these points in Fig. 1 by stars. The $r_{s c}$ and $n_{i}$ dependence (at a fixed $d=5 a_{B}$ ) of the experimental points are in agreement with our calculations, although the range of impurity concentration of the samples of Pudalov et al. ${ }^{2}$ are rather limited.

In summary, we have investigated the phase diagram of a two-dimensional electron system at freezing when charged impurities are present. We have used our improved knowledge of the static response functions in the solid and fluid phases. Our findings are in agreement with recent experimental results.

This work was supported in part by NATO under Grant No. CRG920487. S.T.C thanks the hospitability of Bilkent University Physics Department.
${ }^{1}$ For a recent review, see Physics of the $2 D$ Quantum Electron Solid, edited by S.-T. Chui (International Press, Cambridge, 1994).

${ }^{2}$ V. M. Pudalov, M. D'Iorio, S. V. Kravchenko, and J. W. Campbell, Phys. Rev. Lett. 70, 1866 (1993).

${ }^{3}$ B. Tanatar and D. Ceperley, Phys. Rev. B 39, 5005 (1989).

${ }^{4}$ S. T. Chui and K. Esfarjani, Europhys. Lett. 14, 361 (1991).

${ }^{5}$ S. T. Chui and B. Tanatar, Phys. Rev. Lett. 74, 458 (1995).

${ }^{6}$ S. T. Chui and B. Tanatar, J. Phys. Condens. Matter 7, 5865 (1995)
${ }^{7}$ S. Moroni, D. M. Ceperley, and G. Senatore, Phys. Rev. Lett. 69, 1837 (1992).

${ }^{8}$ L. Bonsall and A. A. Maradudin, Phys. Rev. B 15, 1959 (1977).

${ }^{9}$ K. Esfarjani and S. T. Chui, J. Phys. Condens. Matter 3, 5825 (1991).

${ }^{10}$ N. Iwamoto, Phys. Rev. B 43, 2174 (1991).

${ }^{11}$ Our original calculation (Ref. 6) did not include the response at $r_{s}=10$ in the solid phase.

${ }^{12}$ A. Gold, Phys. Rev. Lett. 54, 1079 (1985). 